\title{
Estrategias de afrontamiento en personas con trastorno mental grave en el contexto laboral
}

\author{
Coping strategies in people with \\ severe mental disorders in the workplace
}

\author{
Carlos Vaquero ${ }^{(1,2)}$, Francisco Ballesteros ${ }^{(1,2)}$, Gema Herradón $^{(1,3)}$, Adriana Sobrino $^{(1,4)}$, Diana \\ Torres $^{(1,5)}$, Mariola Vitutia ${ }^{(1,6)}$, Carlos Molinero ${ }^{(1,7)}$, Alba López $^{(1,2)}$, Vanesa Delgado ${ }^{(1,8)}$, Marina \\ $\operatorname{Larios}^{(1,7)}$, Begoña Gamero ${ }^{(1,3)}$, Eva Maria Muñiz ${ }^{(1,5)}$, Beatriz Esteban ${ }^{(1,5)}$ y Rosana Calvo ${ }^{(1,9)}$ \\ ${ }^{1}$ Centros de Rehabilitación Laboral pertenecientes a la Red Pública de Atención Social \\ a Personas con Trastorno Mental Severo y Duradero de la Comunidad de Madrid. Consejería \\ de Políticas Sociales y Familia de la Comunidad de Madrid, España; \\ ${ }^{2}$ Grupo 5, España; ${ }^{3}$ Fundación Buen Samaritano, España; ${ }^{4}$ Walkredi, España; ${ }^{5}$ Fundación Manantial, España; \\ ${ }^{6}$ Intress, España; ${ }^{7}$ Nueva Vida, España; ${ }^{8}$ Hermanas Hospitalarias del Sagrado Corazón, España; ${ }^{9}$ Grupo Exter, España
}

\begin{abstract}
Resumen: Se trata con este estudio de conocer los estilos de afrontamiento de personas con trastorno mental severo en el entorno laboral. La hipótesis inicial es que las personas de la muestra cuentan con unas estrategias de afrontamiento más ineficaces y/o pasivas que la población general, lo cual podría suponer un riesgo no solo para el mantenimiento del puesto de trabajo, sino para su proceso de recuperación y de integración social. Se trata de un estudio cuantitativo de diseño descriptivo en el que se seleccionó una muestra de 175 personas en situación de empleo atendidas en los Centros de Rehabilitación Laboral (CRL) pertenecientes a la Red Pública de Atención Social a Personas con Enfermedad Mental Severa y Duradera de la Comunidad de Madrid (en adelante la Red). Los instrumentos utilizados son el Inventario de Estrategias de Afrontamiento, CSI y el Cuestionario de Evaluación de Riesgos Psicosociales, DECORE. Los resultados obtenidos indican que las personas que conforman la muestra tienen una percepción de escasa capacidad de resolución de problemas, con tendencia a la evitación de situaciones de conflicto cotidiano, así como a conductas que podrían vincularse al aislamiento o la retirada social. Además suelen mostrarse críticas con su capacidad para resolver situaciones de conflicto cotidianas.
\end{abstract}

\footnotetext{
Todos los autores trabajan, además de en otras instituciones, en Centros de Rehabilitación Laboral pertenecientes a la Red Pública de Atención Social a Personas con Trastorno Mental Severo y Duradero de la Comunidad de Madrid, pertenecientes a la Consejería de Políticas Sociales y Familia de la Comunidad de Madrid.

La correspondencia sobre este artículo debe enviarse al primer autor al Centro de Rehabilitación Laboral Aranjuez C/ Jardinero Boutelou, 75. 28300 Aranjuez.E-mail: cvaquerocrespo@gmail.com
}

(cc) EY-NC-ND Este es un artículo Open Access bajo la licencia CC BY-NC-ND. 
Palabas clave: Estrategias de afrontamiento, trastorno mental, entorno laboral, Centro de Rehabilitación Laboral.

\begin{abstract}
This study addressed the coping skills of people with severe mental disorders in the workplace. The initial hypothesis is that subjects of the sample have coping strategies more ineffective and/or passive than the general population, which may represent a risk for maintenance of the job and for the recovery and social integration process. This was quantitative study of descriptive design that enrolled a sample of 175 employed people who attended the Occupational Rehabilitation Centers (CRL) of the public network for social care to people with severe mental disease of the Madrid regional government. Instruments used for the study included the Coping Strategies Inventory, CSI, and the Questionnaire for Psychosocial Risk Assessment, DECORE. The results suggest that people in the sample have a perception of poor problem-solving capacity, with a trend to avoid situations of daily conflict and to behaviors that could be linked to isolation or social withdrawal. In addition, they usually criticize their own capacity to resolve conflicts in daily life.

Keywords: Coping strategies, mental disorder, workplace, CRL.
\end{abstract}

Históricamente las personas aquejadas de algún problema grave de salud mental son las que tienen mayores tasas de desempleo entre las personas con algún tipo de discapacidad. Según datos de empleo del Instituto Nacional de Empleo (Nota de prensa del INE de 1 diciembre 2017), en 2017 la tasa de empleo de estas personas se situaba en el $15.9 \%$, la más baja de todas las personas registradas con algún tipo de discapacidad reconocida, junto con la de personas con discapacidad intelectual (15.5\%). Si tenemos en cuenta que la consecución de un empleo es, junto con la vivienda y las relaciones sociales, uno de los tres pilares para conseguir la integración comunitaria de las personas con trastornos mentales graves (López, Laviana y González, 2010), nos encontramos ante una situación en la que se hace necesaria una intervención que permita revertir estos datos. Desde hace más de 25 años, los Centros de Rehabilitación Laboral (CRL) tienen entre sus objetivos más relevantes conseguir la inserción laboral y el mantenimiento posterior del empleo de las personas que a estos recursos acuden (Alcaín et al., 2001; Rodríguez, 2006). Los CRL forman parte de la Red Pública de Atención Social a Personas con Trastorno Mental Severo y Duradero de la Comunidad de Madrid. Esta Red está formada por diferentes dispositivos que tratan de lograr la recuperación y participación activa de las personas con estas características en la sociedad. La Red fue creada en la década de los 80 del pasado siglo, en el marco de las políticas de reforma psiquiátrica que incluían la externalización de los hospitales psiquiátricos de las personas con trastorno mental y la integración comunitaria de las mismas. Esta Red cuenta con una amplia tipología de recursos, sin embargo, en el presente artículo, nos centraremos en la población atendida en alguno de los 22 CRL (en el momento del estudio eran 22 CRL, aunque en la actualidad se cuenta con 5 más para llegar a 27 dispositivos de esta índole).

Una de las razones por las que resulta relevante el trabajo de estos centros es, como señalamos antes, la alta tasa de desempleo que afecta a las personas aquejadas de un problema de salud mental grave. Según datos del INE en torno al 85\% de estas personas se encuentra en situación de desempleo y cerca del $65 \%$ no realiza búsqueda del mismo. Nos encontramos entonces ante una situación en la que la persona pierde la oportunidad de, como afirman López, Laviana y González (2010), desarrollarse cognitivamente, disponer de un contexto que organiza la vida cotidiana y que contribuye al desarrollo de habilidades sociales, además de no disponer de ingresos económicos vinculados al trabajo, lo que reduce la valoración y reconocimiento social que un empleo proporciona.

Según Blanco (2010) las persona con trastorno mental tiene un deterioro que se evidencia fundamentalmente en la pérdida de habilidades para el adecuado desempeño de roles sociales. Por tanto, aunque entre las metas que se persiguen en los CRL la principal sea la consecución de un empleo, no resulta menos 
importante la intervención dirigida a tratar de mejorar la capacidad para afrontar situaciones estresantes, desarrollar habilidades de relación que permitan establecer y consolidar interacciones sociales, adquirir habilidades de resolución de problemas y demás competencias que resultan relevantes para lograr el mantenimiento de la inserción laboral. Además de dotar de habilidades, el entrenamiento para adquirir estrategias de afrontamiento eficaces tiene relación con actitudes de afrontamiento más optimistas que redundan en el estado físico de la persona (Martínez, Reyes, García y González, 2006). Por otra parte, la intervención integral desarrollada desde los CRL facilita, no solo la consecución, sino también el mantenimiento de una inserción laboral que pronostica una mejor evolución de los procesos de recuperación de la persona (Naranjo, Cobo, Rebolleda, González y Grupo CRL-LRHP, 2018). Por tanto, la consecución del empleo no pone fin a la intervención, sino que lejos de ser así, suele considerarse un momento especialmente significativo en la misma, puesto que es frecuente que se trate de un periodo de satisfacción por la consecución del empleo, pero también de estrés en el que poner en marcha mecanismos de afrontamiento de situaciones sociolaborales que, bien pueden resultar novedosas en aquellos casos en los que se trata de la consecución de un primer empleo, bien pueden ser conocidas pero no por ello fáciles de gestionar en casos de personas que llevan tiempo sin contacto con el ámbito laboral.

Este estudio forma parte de una investigación más ambiciosa en la que se trata de observar si existe relación entre los riesgos psicosociales y las estrategias de afrontamiento de las personas atendidas en los CRL de la Comunidad de Madrid (CM) (Ballesteros et al., 2019), sin embargo, aquí el objetivo es conocer cuáles son las estrategias de afrontamiento que utilizan las personas con trastorno mental grave atendidas y si pueden considerarse eficaces o no.

\section{Método}

\section{Diseño}

Se trata de una investigación multirecurso de diseño descriptivo transversal de una sola muestra. Se procederá a describir variables sociodemográficas, laborales y clínicas así como los resultados de las estrategias de afrontamiento obtenidas de los resultados del CSI.

\section{Muestra}

Esta estuvo formada por 175 personas, atendidas en alguno de los 22 CRL que formaban parte de la Red de la CM en el momento del estudio (años 2016-2018). La participación tuvo carácter voluntario, las personas firmaron un documento que certificaba su consentimiento informado para participar en la investigación. En la parte descriptiva se recogió información sobre variables sociodemográficas, laborales, clínicas, así como de los estilos de afrontamiento y los riesgos psicosociales.

Entre los criterios de inclusión al estudio se estableció que las personas, debían estar atendidas en alguno de los CRL de la Red que formaban parte del estudio, llevar trabajando 3 meses en el puesto en el que se encontraba y firmar el consentimiento informado. Como criterios de exclusión se estableció no firmar el consentimiento antes mencionado, que la persona sufriera problemas cognitivos que condicionaran la comprensión de las pruebas a administrar y que, en el momento de la recogida de información, la persona estuviera en situación de pluriempleo (dos o más trabajos simultáneos) al considerar estos, factores que podían interferir en la recogida de información y posterior análisis.

La muestra tuvo una mortalidad de un $3.4 \%$ que corresponde a personas que bien no contestaron al Inventario, o bien lo hicieron de forma incompleta. Por tanto, para el análisis de las estrategias de afrontamiento, contamos con 169 personas. 


\section{Variables e instrumentos}

Las variables utilizadas son las que se exponen en la Tabla 1.

Tabla 1. Variables

\begin{tabular}{llll}
\hline Sociodemográficas & Laborales & Clínicas & Estilo afrontamiento \\
\hline Género & Categoría profesional & Diagnóstico & Inventario de estrategias de afron- \\
Edad & Tipo de trabajo & Años de evolución & \\
Nivel educativo & Tiempo en el trabajo actual & Baja laboral & \\
& Tipo de jornada & Patología asociada & \\
& Tipo de salario & \\
& Sueldo & \\
& Tipo de contrato & \\
& Tipo de empresa & \\
\hline
\end{tabular}

Para la recogida de datos se utilizaron dos pruebas validadas en castellano. Por una parte el Inventario de Estrategias de Afrontamiento de Tobin, Holroyd, Reynolds y Kigal, 1989; adaptado por Cano, Rodríguez y García en 2006, que explora la eficacia del afrontamiento ante situaciones de estrés mediante ocho dimensiones: Resolución de problemas (REP), Autocrítica (AUT), Expresión de emociones (EEM), Pensamiento desiderativo (PSD), Apoyo social (APS), Reestructuración cognitiva (REC), Evitación de problemas (EVP) y Retirada social (RES).

Esta herramienta está formada por 41 ítems que se agrupan en ocho factores de los cuales cuatro hacen referencia a estrategias ineficaces de afrontamiento, (AUT, PSD, EVP y RES), mientras que los otros cuatro recogen aspectos eficaces de afrontamiento, (REP, EEM, REC y APS). El afrontamiento eficaz se corresponde con unas puntuaciones bajas en los cuatro primeros factores y elevadas en los últimos. Por otra parte, de los ocho factores, hay cuatro que se relacionan con aspectos cognitivos (AUT, PSD, REP y REC) y otros cuatro con aspectos conductuales (EVP, RES, EEM y APS).

En el estudio completo, se utilizó también el Cuestionario de Evaluación de Riesgos Psicosociales DECORE de Luceño y Martín (2008). Esta herramienta evalúa la percepción de riesgos psicosociales y la relación que estos tienen con el estrés o el riesgo de padecer alguna enfermedad asociada al trabajo. Distingue cuatro escalas: Control, Recompensas, Apoyo organizacional y Demandas cognitivas. Además incorpora tres índices que se obtienen de la combinación de los diferentes riesgos psicosociales evaluados y que son: índice de desequilibrio demanda-control, índice de desequilibrio demanda-recompensa e índice global de riesgo. No obstante, los resultados obtenidos en este cuestionario serán objeto de análisis en otro artículo.

Los cuestionarios fueron administrados por los/as psicólogo/as de los CRL o por otros profesionales de los CRL entrenados ad hoc para la tarea.

\section{Análisis de datos}

Se han realizado análisis descriptivos y de frecuencias de las variables sociodemográficas, laborales y clínicas objeto de estudio, así como el análisis de los resultados en la variable de afrontamiento mediante los resultados del CSI. Los datos obtenidos en esta prueba, provienen de la transformación de las puntuaciones directas de cada individuo en una media global para cada factor. Estas se han obtenido posteriormente en 
centiles utilizando para ello los baremos del CSI. De esta manera se ha obtenido una única puntuación para cada factor del CSI, a partir del cual se han extraído las conclusiones. Para comparación interfactorial del CSI se utilizó el Coeficiente de correlación de Pearson. La comparación de grupos en las variables de mayor interés se realizó mediante t de Student y ANOVA. Debido al tamaño de la muestra se asume la normalidad. El programa informático utilizado fue el SPSS versión 22.

\section{Resultados}

En las tablas que aparecen a continuación se recogen las características sociodemográficas de la muestra. Encontramos un porcentaje significativamente mayor de hombres que de mujeres. La mayoría de las personas que forman parte de la muestra (65.7\%) tienen edades comprendidas entre los 31-45 años.

La Tabla 2 muestra que el diagnóstico más habitual (59.4\%) es el de esquizofrenia u otro trastorno del espectro psicótico.

Tabla 2. Distribución de muestra por diagnóstico

\begin{tabular}{ll}
\hline Diagnóstico & Categoría (\%) \\
\hline Esquizofrenia & $45.7 \%$ \\
\hline Trastorno psicótico & $13.7 \%$ \\
\hline Trastorno del estado de ánimo & $13.1 \%$ \\
\hline Trastorno de personalidad & $9.1 \%$ \\
\hline Trastornos de ansiedad & $2.9 \%$ \\
\hline Otros trastornos & $8.0 \%$ \\
\hline Sin especificar & $6.9 \%$ \\
\hline
\end{tabular}

En la Tabla 3 se observa que más del 50\% de las personas poseen estudios secundarios, lo que supone un porcentaje importante de personas con, al menos, formación de nivel medio.

Respecto a las características laborales de la muestra, en su mayoría se trata de personas que trabajan en "empleo protegido" al estar contratados por Centros Especiales de Empleo (CEE). Sin embargo, una parte importante de ellas lo hace en enclaves laborales que suponen el desarrollo de su trabajo en un entorno ordinario, aunque dependan administrativamente de un CEE.

Desarrollan su labor en puestos de baja cualificación profesional, fundamentalmente como auxiliares o peones.

La Tabla 4 ofrece información sobre el rango salarial. El salario medio se sitúa por debajo de los $600 €$ en más del $85 \%$ de los casos. Aunque en todos los casos se alcanza el Salario Mínimo Interprofesional (SMI), ajustado al porcentaje de jornada, solo un porcentaje del 5.7\% de personas obtiene salarios superiores al SMI en el momento del estudio. 
Tabla 3. Distribución de la muestra por categoría profesional

\begin{tabular}{ll}
\hline Categoría profesional & Categoría (\%) \\
\hline Ingenieros y licenciados & $1.1 \%$ \\
\hline Ingenieros técnicos, peritos, Ayudantes titulados & $1.1 \%$ \\
\hline Jefes administrativos & $0.6 \%$ \\
\hline Ayudantes no titulados & $10.3 \%$ \\
\hline Oficiales administrativos & $2.9 \%$ \\
\hline Subalternos & $4.6 \%$ \\
\hline Auxiliares administrativos & $21.7 \%$ \\
\hline Oficiales de primera & $2.9 \%$ \\
\hline Oficiales de tercera & $4.6 \%$ \\
\hline Peones & $49.1 \%$ \\
\hline
\end{tabular}

Tabla 4. Distribución de la muestra por rango salarial

\begin{tabular}{cc}
\hline Rango Salarial & Categoría (\%) \\
\hline $300 €$ o menos & $11.4 \%$ \\
\hline $301-450 €$ & $28.6 \%$ \\
\hline $451-600 €$ & $45.1 \%$ \\
\hline $601-750 €$ & $8.6 \%$ \\
\hline $751-900 €$ & $4.0 \%$ \\
\hline $901-1200 €$ & $1.1 \%$ \\
\hline $1501-1800 €$ & $0.6 \%$ \\
\hline
\end{tabular}


La tabla 5 hace referencia al tipo de jornada. Las condiciones de trabajo indican que se suele cumplir con los horarios y jornadas establecidas y no resulta frecuente la realización de horas extraordinarias. Esto no solo puede suponer un factor de protección frente a posibles estresores laborales por incremento de jornada laboral, sino que además es un indicador de calidad en el empleo como se recoge en el Real Decreto-ley 8/2019, de 8 de marzo, de medidas urgentes de protección social y de lucha contra la precariedad laboral en la jornada de trabajo que entró en vigor el domingo 12 de mayo de dicho año.

\section{Tabla 5. Distribución de la muestra por tipo de jornada laboral ( $\mathbf{N}^{0}$ de horas/semana)}

\begin{tabular}{cc}
\hline $\mathbf{N}^{\mathbf{0}}$ de horas/semana & Categoría $(\%)$ \\
\hline 30 horas menos & $45.7 \%$ \\
\hline $31-35$ horas & $22.3 \%$ \\
\hline $36-40$ horas & $26.3 \%$ \\
\hline $41-45$ horas & $4.6 \%$ \\
\hline más de 45 horas & $0.6 \%$ \\
\hline
\end{tabular}

Otros datos obtenidos indican que el tiempo de permanencia en la misma empresa supera los 2 años en el $45.5 \%$ de la muestra, incidiendo de manera clara en la fidelidad laboral por parte de la persona y la empresa.

La tipología de contratos se distribuye de manera casi equitativa entre contratos temporales (52\%) y estables $(48 \%)$. Sin embargo, según datos del INE, esto no resiste una comparativa con la población general de asalariados, entre quienes el porcentaje de contratación indefinida, no baja del 73.9\% durante 2016.

Los datos indican que las personas de la muestra permanecen contratadas en la misma categoría profesional desde que comienzan a trabajar para la empresa, lo que sugiere una escasa promoción hacia puestos de mayor cualificación o responsabilidad un factor que podría generar cierto malestar o sensación de estancamiento laboral. La mitad de la muestra tiene la posibilidad de ausentarse del trabajo para ocuparse de cuestiones personales (citas médicas, etc...), a pesar de que, como viene recogido en el convenio que regula los CEE, todas las personas contratadas en este tipo de empresas disponen de un número de días (20 días) para acudir a citas relativas a su situación médica o psicosocial.

Existen pequeñas diferencias, aunque estas son significativas, en cuanto a las estrategias de afrontamiento por cuestiones de género en la escalas del CSI RES $(t=2.023, g l=167, p=.045)$ y en la escala CSI PSD $(t=$ 2.169, $g l=167, p=.032$ ) en las que los hombres obtienen mejores puntuaciones.

La tabla 6 ofrece los resultados del inventario CSI. Un dato que llama la atención es la elevada Desviación típica que muestran todos los factores. Esto parece asociado a una limitación del propio instrumento, puesto que es similar a las desviaciones que se observan en los baremos que proporciona el inventario, indicando por tanto una elevada dispersión de la muestra. Una vez obtenido el perfil, podemos hablar de una baja percepción de la capacidad para resolver situaciones cotidianas $(\mathrm{REP}=35)$, con tendencia a las rumiaciones autoreferenciales de carácter negativo (AUT $=65$ ). Esto no tiene por qué estar relacionado necesariamente con el significado psicopatológico del término. Lo que puede indicar este dato es que las personas evaluadas tienden a valorar peor su capacidad para resolver situaciones cotidianas, es decir, se sienten menos capaces de resolver conflictos de una forma adaptativa. Podríamos vincular este resultado con la evidente dificultad que las personas con problemas mentales graves tienen para enfrentarse a situaciones de estrés, como en este caso las laborales, pero 
también a los conflictos personales, familiares o aquellas situaciones que requieren de la puesta en marcha de habilidades de relación que en gran parte de las personas atendidas en los CRL se muestran deficitarias. Este déficit podría ser causa y a la vez consecuencia del problema de salud mental, por lo que resulta necesaria una intervención que reduzca la incidencia de estas cuestiones en la vida cotidiana de las personas que se atienden en los centros.

Además parece claro que el perfil refleja una tendencia evitativa tanto a nivel cognitivo como conductual como muestran los centiles altos de 70 y 80 en EVP y RES respectivamente. El factor evitativo que parece más asociado a aspectos cognitivos, no muestra puntuaciones elevadas ( $\mathrm{PSD}=45)$. Esto indica la presencia de comportamientos de huida que no parecen estar directamente relacionados con pensamientos similares, sino que podrían tener que ver con los pensamientos autocríticos a los que antes nos referíamos.

\section{Tabla 6. Resultados Inventario estrategias de afrontamiento (CSI)}

\begin{tabular}{llll}
\hline CSI & Media & DT & Puntuación centil \\
\hline REP & 13.17 & 4.99 & 35 \\
\hline AUT & 6.98 & 6.12 & 65 \\
\hline EEM & 8.75 & 5.27 & 50 \\
\hline PSD & 12.71 & 5.29 & 45 \\
\hline APS & 10.54 & 5.51 & 45 \\
\hline REC & 10.75 & 5.18 & 50 \\
\hline EVP & 8.64 & 4.96 & 70 \\
\hline RES & 7.51 & 5.10 & 80 \\
\hline
\end{tabular}

En la Tabla 7 aparecen las correlaciones interfactoriales en la que pueden observarse puntuaciones significativas intermedias entre los pares PSD-AUT, RES-AUT EVP-REC, APS-EEM, $(p=0,01)$. Aunque hay otras relaciones significativas, estas no son muy elevadas (por debajo de .40).

\section{Discusión y Conclusiones}

¿Sería razonable pensar que el perfil evitativo encontrado en la muestra puede estar relacionado con la vulnerabilidad al estrés? ¿Y con las relaciones de apego? Si tomamos como referencia la teoría de vulnerabilidad al estrés Zubin y Spring (1977), podríamos responder de manera afirmativa a la primera pregunta, puesto que estos autores propusieron, hace ya más de 40 años, que las personas con problemas severos de salud mental tienen especial dificultad para gestionar situaciones de estrés debido a que ven superada su capacidad de afrontamiento. En el caso de esta muestra, la capacidad se ve superada no tanto en el ámbito cognitivo, como a la hora de poner en práctica las posibles alternativas que puedan valorar. Sin embargo, no se trata de una 
Tabla 7. Correlaciones interfactoriales

\begin{tabular}{|c|c|c|c|c|c|c|c|c|}
\hline Factores & REP & AUT & EEM & PSD & APS & REC & EVP & RES \\
\hline REP & 1 & & & & & & & \\
\hline AUT & .038 & 1 & & & & & & \\
\hline EEM & $.181 *$ & $.280 * *$ & 1 & & & & & \\
\hline PSD & .051 & $.460 * *$ & $.369 * *$ & 1 & & & & \\
\hline APS & $.236 * *$ & $.157 *$ & $.579 * *$ & $.292 * *$ & 1 & & & \\
\hline REC & $.393 * *$ & .122 & $.271 * *$ & .079 & $.364 * *$ & 1 & & \\
\hline EVP & $.174 *$ & .082 & .078 & .054 & .083 & $.509 * *$ & 1 & \\
\hline RES & -.093 & $.509 * *$ & .053 & $.348 * *$ & -.108 & .030 & $.300 * *$ & 1 \\
\hline
\end{tabular}

* La correlación es significativa en el nivel .05 (2 colas).

** La correlación es significativa en el nivel .01 (2 colas).

característica genotípica en la que el componente biológico, hereditario o inmutable del que hoy seguimos sin noticias, condicione la respuesta de la persona ante la situación de conflicto, sino que más bien, como afirman Bentall y Dillon (2016), parece estar asociado a procesos de aprendizaje de situaciones problemáticas previas. En un porcentaje elevado entre las personas que forman la muestra, se pueden encontrar ejemplos de situaciones vitales traumáticas que han condicionado o en el peor de los casos anulado, la capacidad de identificar y gestionar emociones que interfieren en la toma de decisiones, no solo porque no se dispusiera de dichas habilidades, sino también porque estas se vieron superadas por la situación. Esto nos llevaría a responder a la segunda pregunta también de manera afirmativa, puesto que, la dificultad para gestionar emociones de manera adaptativa o establecer relaciones afectivas sanas en la vida adulta, parece tener relación con las dificultades en el establecimiento de los vínculos de apego aprendidos durante los primeros años de vida (Barroso, 2011). $\mathrm{Si}$ estas relaciones de apego se corresponden con el apego inseguro, ya sea este evitativo o ambivalente (Oliva, 2014) es habitual que se produzcan conductas evitativas o de afrontamiento ineficaz que hayan arraigado como formas de relación que se evidencian en situaciones de interacción social. Por tanto, es posible que las vivencias relacionales durante la etapa infantil generen procesos de aprendizaje que, de consolidarse, tienden a repetirse a lo largo de la vida adulta. Podemos pensar que además de que estas tendencias evitativas estén relacionadas con la dificultad para afrontar y resolver de manera adaptativa situaciones potencialmente estresantes, se evite el conflicto de una manera aprendida tras experiencias traumáticas previas en la historia vital de las personas en momentos en los que se deberían desarrollar estas cuestiones. Podríamos estar hablando entonces de una experiencia de "fracaso" a la que en muchas ocasiones, en la vida adulta, se suman situaciones de sobreprotección del entorno que refuerza conductas evitativas (sobre todo en familias y/o profesionales con tendencia a la sobreimplicación emocional).

Desde los CRL se trabaja para que la persona sea capaz de desarrollar alternativas y habilidades para afrontar las situaciones estresantes cotidianas con éxito. Aunque parece que, en parte, se consigue que genere dichas herramientas como indica la puntuación centil de 50 en REC, no está tan claro que pueda ponerlas en práctica de 
manera habitual. Por nuestra experiencia y los resultados obtenidos, encontramos que no suele ser habitual que la persona llegue a compartir sus ideas acerca de la posible solución de situaciones problemáticas con otros, de manera que reciban feedback sobre la conveniencia o no de las mismas. Para eso, sería necesario un contacto social satisfactorio o eficaz que, según parece en la muestra analizada, se situa en puntuaciones intermedias cuando lo eficaz sería que se encontraran puntuaciones elevadas (APS = 45 y EEM = 45).

Se podría concluir, por tanto, que la baja percepción en la capacidad para resolver problemas $(\mathrm{REP}=35)$ puede estar relacionada con la tendencia a evitar los mismos $(E V P=70)$ y a conductas pasivas y evitativas cuando estos se presentan (RES $=80$ ). Sería interesante conocer respecto a esta "Retirada Social", con qué red de apoyos cuenta la persona, puesto que la experiencia nos muestra que es frecuente un déficit en la cantidad y calidad de apoyos sociales y familiares saludables. Por otra parte, es razonable pensar que la retirada social y aislamiento que se produce en muchos casos con la aparición de la enfermedad, puede dejar una huella en forma de hábito que se reproduce al tener que enfrentarse a situaciones sociolaborales estresantes.

Señalar como limitación este estudio la no aleatorización de la muestra, pero se ha de tener en cuenta que se ha tratado de llegar a casi la totalidad de la población atendida en los CRL que se encontraba trabajando en el momento de la investigación.

La intervención desde los centros de rehabilitación laboral está focalizada en la persona para la adquisición o recuperación de competencias sociolaborales que le permitan conseguir una inserción laboral y su posterior mantenimiento, sin embargo, no se debe olvidar la importancia de continuar trabajando con el tejido empresarial para sensibilizar y lograr oportunidades de empleo que permitan mantener un equilibrio entre la rentabilidad económica y la creación de empleo estable de calidad (López, Álvarez y Laviana, 1998) que favorezca la generación de entornos laborales saludables. Esto supone una perspectiva de intervención comunitaria que entiende las empresas como entornos de intervención y no solo de uso.

\section{Referencias}

Alcaín, E., Colis, J. A., Galilea, V. Lavado, A. I., Muñiz, E. Nicolás, M., ... Sobrino, T. (2001). Rehabilitación laboral de personas con enfermedad mental crónica: Programas básicos de intervención. (Cuadernos técnicos de servicios sociales, 14). Madrid, España: Consejería de Servicios Sociales.

Ballesteros F., Vaquero C., Vitutia M., Molinero C., Larios M., Delgado V., ... Calvo R. (2019). Análisis de la percepción de riesgos psicosociales en personas con trastorno mental grave en el contexto laboral. Clínica Contemporánea, 10, e11. https://doi.org/10.5093/cc2019a11

Barroso, O. (2014). El apego adulto: la relación de los estilos de apego desarrollados en la infancia en la elección y las dinámicas de pareja. Revista Digital de Medicina Psicosomática y Psicoterapia. 4(1), 1-25.

Bentall, R. y Dillon, J. (2016). Modelos de locura II. Barcelona, España: Editorial Herder.

Blanco, A. (2010). El enfermo mental con discapacidades psicosociales. En A. Pastor, A. Blanco y D. Navarro. (Coords.). Manual de rehabilitación del trastorno mental grave (pp. 77-94). Madrid, España: Síntesis.

Cano, F. J., Rodríguez, L. y García, J. (2007). Adaptación española del Inventario de Estrategias de Afrontamiento. Actas Españolas de Psiquiatría. 35(1), 29-39.

Instituto Nacional de Estadística (INE). (1 de diciembre de 2017). El Empleo de las Personas con Discapacidad. Año 2016. [Notas de Prensa]. Recuperado de https://www.ine.es/prensa/epd 2016.pdf

López, M., Álvarez, F. y Laviana, M. (1998). Empresas sociales y rehabilitación psiquiátrica en Andalucía. Revista de la Asociación Española de Neuropsiquiatría. 18, 735-747.

López, M., Laviana, M. y González, S. (2010). Rehabilitación laboral y programas de empleo. En A. Pastor, A. Blanco y D. Navarro (2010). (Dirs.). Manual de rehabilitación del trastorno mental grave (pp. 511-537). Madrid, España: Síntesis.

Luceño, L. y Martín, J. (2008). DECORE. Cuestionario de evaluación de riesgos psicosociales. Madrid, España: TEA Ediciones.

Martínez, A., Reyes, G. A, García, A. y González, M. I. (2006). Optimismo/pesimismo disposicional y estrategias de afrontamiento del estrés. Psicothema 18, 66-72. 
Naranjo, R., Cobo, F., Rebolleda, C., Gonzalez E. y Grupo CRL-LRHP (2018). Los centros de rehabilitación laboral y el trastorno mental grave: La experiencia y el seguimiento de los usuarios en la Comunidad de Madrid. Psychosocial Intervention 27, 65-71.

Oliva, A. (2004). Estado actual de la teoría del apego. Revista de Psiquiatría y Psicología del niño y del adolescente, 4(1), 65-81.

Real Decreto-Ley 8/2019, de 8 de marzo, de medidas urgentes de protección social y de lucha contra la precariedad laboral en la jornada de trabajo. Boletín Oficial del Estado (España), 61, de 12 de marzo de 2019. Recuperado de https://www.boe.es/boe/dias/2019/03/12/pdfs/BOE-A-2019-3481.pdf

Rodríguez, A. (Coord). (2006). Rehabilitación psicosocial de personas con trastornos mentales crónicos. Madrid, España. Pirámide.

Zubin, J. y Spring, B. (1977). Vulnerability: A new view of schizophrenia. Journal of Abnormal Psychology, 86(2), 103-126.

Artículo recibido: 01/08/2019

Artículo aceptado: 20/10/2019 